\title{
The Role of Activated Microglia and Resident Macrophages in the Neurovascular Unit during Cerebral Ischemia: Is the Jury Still Out?
}

\author{
Rawan Barakat Zoran Redzic \\ Department of Physiology, Faculty of Medicine, Kuwait University, Kuwait City, Kuwait
}

\begin{abstract}
Key Words
Blood-brain barrier . Cerebral ischemia - Cytokines .

Macrophages $\cdot$ Microglia $\cdot$ Stroke
\end{abstract}

\begin{abstract}
Paracrine signaling in the neurovascular unit (NVU) is aimed to adjust the supply of oxygen and nutrients to metabolic demands of the brain in a feed-forward manner. Cerebral ischemia $(\mathrm{Cl})$ severely disrupts this homeostatic mechanism and also causes activation of microglia and resident macrophages in the brain. Contradictory data exist on the time pattern of microglial activation and polarization during $\mathrm{Cl}$, on molecular mechanisms that trigger them and on effects of microglia-derived cytokines on brain cells. It appears that conditions that occur during transient ischemia or in the penumbra of focal ischemia in vivo or equivalent conditions in vitro trigger polarization of resting microglia/macrophages into the $M 2$ phenotype, which mainly exerts antiinflammatory and protective effects in the brain, while prolonged ischemia with abundant necrosis promotes microglial polarization into the $\mathrm{M} 1$ phenotype. During the later stages of recovery, microglia that polarized initially into the M2 phenotype can shift into the M1 phenotype. Thus, it appears that cells with both phenotypes are present in the affected area, but their relative amount changes in time and probably depends on the proximity to the ischemic core. It was assumed that cells with the M1 phenotype exert detri-
\end{abstract}

mental effects on neurons and contribute to the blood-brain barrier opening. Several M1 phenotype-specific cytokines exert protective effects on astrocytes, which could be important for reactive gliosis occurring after ischemia. Thus, whether or not suppression of microglial activity after $\mathrm{Cl}$ is beneficial for neurological outcome still remains unclear and current evidence suggests that no simple answer could be given to this question.

(c) 2015 S. Karger AG, Basel

\section{Brain Cells Are Functionally Integrated into the Neurovascular Unit}

The concept of the neurovascular unit (NVU) was introduced in 2001 by the National Institute of Neurological Disorders and Stroke (US National Institutes of Health); it defined NVU as a functional unit consisting of neurons, astrocytes, brain endothelial cells (BECs) with basal lamina matrix, pericytes, vascular smooth muscle cells, microglia and oligodendrocytes (fig. 1; animation for the figures was produced by R.B. using tools from Servier Medical art: http://www.igis.com/medicalimages/?slidekit=35) [1,2]. Functional interaction of NVU cells through bidirectional cell-cell signaling provides optimal adjustment of the brain vasculature, including cerebral blood flow (CBF) and capillary permeability to match metabolic demands [1-6]. Under normal

\begin{tabular}{ll}
\hline KARGER & $\begin{array}{l}\text { () 2015 S. Karger AG, Basel } \\
1011-7571 / 15 / 0257-0003 \$ 39.50 / 0 \quad \text { Karger }\end{array}$ \\
$\begin{array}{l}\text { E-Mail karger@karger.com } \\
\text { www.karger.com/mpp }\end{array}$ & $\begin{array}{l}\text { This is an Open Access article licensed under the terms of the } \\
\text { Creative Commons Attribution-NonCommercial 3.0 Un- } \\
\text { ported license (CC BY-NC) (www.karger.com/OA-license), } \\
\text { applicable to the online version of the article only. Distribu- } \\
\text { tion permitted for non-commercial purposes only. }\end{array}$
\end{tabular}

Dr. Zoran Redzic

Department of Physiology, Faculty of Medicine, Kuwait University PO Box 24923

Safat 13110 (Kuwait)

E-Mail redzic@ hsc.edu.kw 
Fig. 1. The neurovascular unit (NVU): a key role of the NVU is to facilitate adjustments of local blood flow and the permeability of capillaries to neuronal activity in a feed-forward manner. An important factor that regulates local blood flow in the brain is the contractility of pericytes; both pericytes and endothelial cells come in close contact with end feet of astrocytes; the latter cells play a key role in neurovascular coupling because they are in close proximity to neuronal synapses (thus, have paracrine signaling from these cells) on one side and have end feet that come in close contact with endothelial cells and pericytes on the other side, which permits paracrine and direct cell-to-cell communication. Microglia survey the microenvironment, though a precise role of these cells on brain capillaries in physiological conditions is not well established yet.

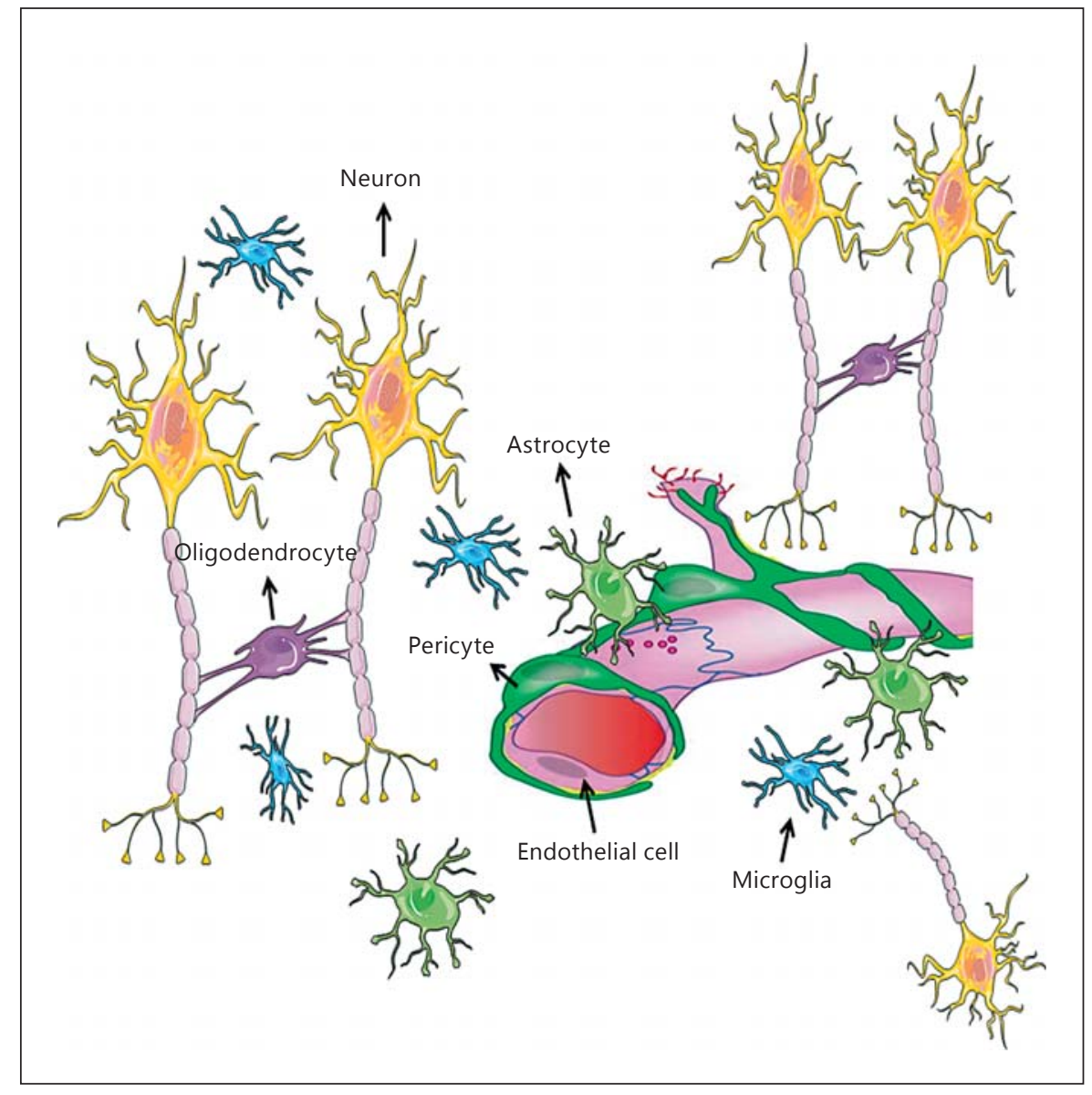

conditions, $\mathrm{CBF}$ in humans remains $>50 \mathrm{ml} / \mathrm{min} / 100 \mathrm{~g}$ brain tissue and its regional variations reflect adjustments to metabolic demands of a particular region. These adjustments are mainly triggered by the release of glutamate, the most abundant excitatory neurotransmitter in the brain $[7,8]$, from neurons that bind to N-methyl-Daspartate receptor (NMDA) and metabotropic glutamate receptors (MGluR) in neurons and astrocytes, respectively [7, 8] (fig. 2). Glutamate-mediated signaling leads to the release of nitric oxide (NO) from neurons and the production of arachidonic acid (AA) in astrocytes. AA is quickly metabolized to prostaglandin $\mathrm{E}_{2}$ and epoxyeicosatrienoic acids $[7,9,10]$ that cause a reduction in $\mathrm{Ca}^{2+}$ concentration in vascular smooth muscle cells and in pericytes, thereby relaxing contractile filaments, which results in vasodilation and, hence, in an increase in $\mathrm{CBF}$ [7] (fig. 2). In addition, a rise in $\mathrm{Ca}^{2+}$ in astrocytes due to glutamate-mediated signaling results in stimulation of large-conductance $\mathrm{Ca}^{2+}$-activated $\mathrm{K}^{+}(\mathrm{BK})$ channels in astrocyte end feet and release of $\mathrm{K}^{+}$in the extracellular space close to cerebral microvessels (fig. 2) [7, 11]. An increase in $\mathrm{K}^{+}$concentration (up to $10 \mathrm{mM}$ ) hyperpolarizes smooth muscle cells, which reduces the influx of $\mathrm{Ca}^{2+}$ through voltage-gated channels and, hence, causes dilatation of blood vessels [7].

A rise in $\left[\mathrm{Ca}^{2+}\right]_{\mathrm{i}}$ in astrocytes causes constriction of cerebral arterioles, which reduces CBF. This is mediated by metabolic transformation of astrocyte-derived AA into 20-hydroxy-eicosatetraenoic acid, a process that occurs in vascular smooth muscle cells $[7,9,10,12]$ (fig. 2).

Whether a rise in astrocyte $\left[\mathrm{Ca}^{2+}\right]_{\mathrm{i}}$ causes dilation or constriction of an arteriole may in part be determined by the preexisting tone of the vessel [13], but also by partial pressure of $\mathrm{O}_{2}$ in the tissue [7]. Astrocytes also synthesize and release various vasoactive substances, such as prostacyclin, adenosine and adenosine triphosphate (ATP), that affect the tonus of vascular smooth muscle cells [14], but these signaling molecules control vascular tone in a feedback rather than in a feed-forward manner (fig. 2). 


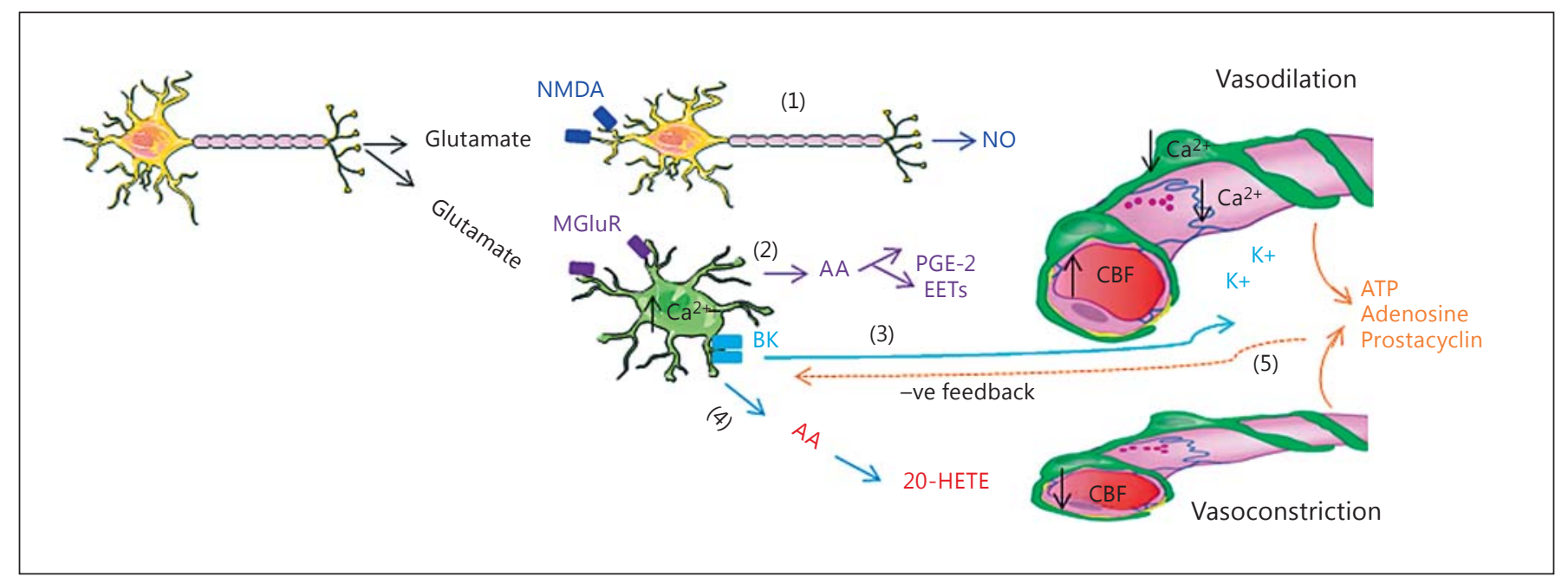

Fig. 2. A simplified diagram of paracrine signaling in the neurovascular unit. Neuronal activity (i.e. glutamate release) induces vasodilation through its action on interneurons or astrocytes. As a response to this stimulation, interneurons release NO (1), while astrocytes induce vasodilation through several mechanisms $(2,3)$. In addition to that, astrocyte-derived AA (4) could be metabolized into 20-hydroxy-eicosatetraenoic acid (20-HETE) in smooth muscle cells, which then induces vasoconstriction. Signaling from capillaries affects the tone of vascular smooth muscle cells via astrocytes (5). PGE-2 = Prostaglandin $\mathrm{E}_{2}$; EETs = epoxyeicosatrienoic acids.
Brain microenvironment is largely regulated by the permeability of brain capillaries, which have a unique phenotype that severely limits free diffusion of solutes and molecules across the endothelial layer. This unique phenotype is a morphological substrate for the so-called blood-brain barrier (BBB). Functionally, the BBB consists not only of BECs but is rather a complex cellular system with several cell types and basal lamina components which regulate its function $[6,15]$. A large number of pericytes are embedded in the basal membrane that surrounds microvessels, and astrocytic end feet are in close proximity. Astrocytic end feet and BECs interact to form the interendothelial tight junctions and basal lamina [2, $3,16]$. Pericytes are distributed discontinuously along the length of the cerebral capillaries and partially surround the endothelium. Both endothelial cells and pericytes produce and are enclosed by a perivascular extracellular matrix [16].

The influence of astrocytes appears to be essential to induce the tight junction formation in BECs through paracrine signaling mediated by several trophic and growth factors $[4,16]$. In hypoxic conditions, astrocytes produce vascular endothelial growth factor (VEGF), which is a key signaling molecule that triggers BEC migration and proliferation [17]. On the other hand, BECs release leukemia inhibitory factor, which affects the differentiation and maturation of astrocytes $[18,19]$. Pericytes contribute to the regulation of BBB integrity by playing a role in the induction of occludin expression, which is one of the major tight junction proteins $[4,15]$. Beside their primary role in adjusting capillary diameter to metabolic demands, pericytes could also affect BEC differentiation and phenotype via growth factor release, which stimulates endothelial cell proliferation and differentiation, and angiotensin 1 , which plays a role in endothelial cell maturation and in the induction of their specific phenotype. On the other hand, endothelial cells secrete platelet-derived growth factor- $\beta$, which stimulates the recruitment of pericytes to newly formed vessels [5, 20-22]. Microglia are also closely associated with the endothelium and play supporting roles in the BBB induction and maintenance. It appears that microglia can affect remodeling of cerebral vessels, enhance vessel branching in the mature brain and activate angiogenesis $[23,24]$. Under normal conditions, microglia secrete transforming growth factor (TGF)- $\beta$, which inhibits endothelial cell proliferation, thus stabilizing microvascular permeability [25].

\section{Microglia Play an Important Role in the NVU}

Microglia originate from the yolk sac and populate the central nervous system (CNS) early in the development as amoeboid cells [26]. They are present more or less 
evenly in all regions of the brain and account for about $20 \%$ of the glial population in the brain [27]. Under physiological conditions after birth, microglia are in the resting state, which is characterized by a ramified phenotype with little cytoplasm [28]. These cells are dynamic; they continuously extend and retract their processes, surveying the microenvironment [28-30]. During development, microglia play an important role in neuronal migration, axonal growth and synaptic remodeling [27, 28]. During postnatal development, microglia are responsible for phagocytosis of immature synapses [28]. However, the main functions of microglia are pathogen recognition, phagocytosis of damaged/apoptotic cells, scavenging of the damaged neurons, small and inactive synapses, tissue debris, infectious agents and macromolecules, regulation of T-cell responses and induction of inflammation [28, 31-34]. Beside these roles, microglia actively interact with other NVU cells, and this interaction plays an important role in keeping the environment stable and in maintaining hemostasis, as described above.

Communication of microglia with other cells of the $\mathrm{NVU}$ is achieved mainly by paracrine signaling that is mediated by cytokines, chemokines and growth factors [33, 35, 36]. Microglia can affect neuronal activity by releasing ATP, which then stimulates astrocytes to release glutamate, which in turn binds to neuronal mGluR5, thereby increasing excitatory postsynaptic current frequency $[28$, 37]. In addition, microglia play a role in establishing functional neuronal circuits through several paracrine mechanisms that involve the fractalkine receptor (also known as G-protein-coupled receptor-13 or GPR13), complement receptors, interleukin (IL)-1 $\beta$ and adaptor protein (DAP12) $[28,38]$. Connections between neurons can be modified by microglia-derived cytokines, proteases and neurotrophic factors, which regulate gap junctions and synapses [34]. On the other hand, it appears that a direct physical interaction between microglia with neurons and astrocytes could also affect neuronal excitability [38].

Communication between microglia and neurons is bidirectional and neurons can also modulate microglia [38, 39]. They can induce and maintain a quiescent (resting) state of microglia by activation of ligand receptors pairs (CD200-CD200R), chemokine C-X3-C motif ligand-1 (CX3CL1-CX3CR1) and by actions of GABA that is released from inhibitory interneurons $[38,40]$. Electrical activity of neurons and neurotrophin release could also maintain microglia in the resting state [31, 39]. In contrast, release of nucleotides such as ATP and excessive glutamate release can activate microglia $[28,38,40]$. It is not clear to which extend this signaling is important for microglial activation in pathological conditions, including CI or hypoxia in the brain.

Microglia are also influenced by astrocytes. During normal physiological conditions, astrocytes inhibit microglial activation by activating soluble astrocyte-derived factors such as nuclear factor (erythroid-derived 2)-like 2 (Nrf2) in microglia, which decreases the microglial production of antioxidant enzymes and, thus, also microglial reactive oxygen/nitrogen species production [41]. Astrocytes also release TGF- $\beta$, macrophage colony-stimulating factors and granulocyte/macrophage colony-stimulating factors which induce ramification [8]. In addition, astrocytes suppress the microglial expression of IL-12 and the production of inducible NO synthase, mediators that induce inflammation $[8,31]$. It is likely that these pathways could play some role in microglia activation during CI.

\section{NVU during $\mathrm{Cl}$}

$\mathrm{CI}$ occurs as a consequence of a reduction in the CBF to a point that supply of oxygen cannot meet metabolic demands. As a consequence, functional impairment and/ or cell death occur, which has been extensively reviewed [42-45]. After the onset of CI, the function and structure of neurovascular coupling become affected or disrupted [46]. Prolyl-hydroxylase activity enhances hypoxia-inducible factor-triggered paracrine signaling in the NVU that is mainly aimed to provide neovascularization and, hence, better perfusion of the tissue; this is an expected response of any tissue to hypoxia [47]. In the brain, this process includes degradation of the vascular matrix and dedifferentiation of BECs, with loss of their typical features (fig. 3); these processes affect BBB integrity [48, 49]. It is assumed that loss of $\mathrm{BBB}$ integrity (or the so-called secondary 'opening' of the BBB) could play a role in exacerbation of neuronal injury via (a) plasma-borne molecules that normally could not enter interstitial fluid of the brain and that could interfere with neurotransmission; (b) extravasation of plasma proteins, and (c) net flux of ions (mainly $\mathrm{Na}^{+}$and $\mathrm{Cl}^{-}$, but probably also $\mathrm{K}^{+}$) into the brain intracellular fluid [50], a process that does not occur under normal conditions. The latter two events contribute to the development of cerebral edema [48], while presence of plasma proteins in the interstitial fluid could contribute to microglial activation.

Several mechanisms that play a role in neovascularization are involved in the development of BBB dysfunction. First, a dysregulation of the activity of the extracellular matrix metalloproteinase (MMP) family occurs. Mi- 


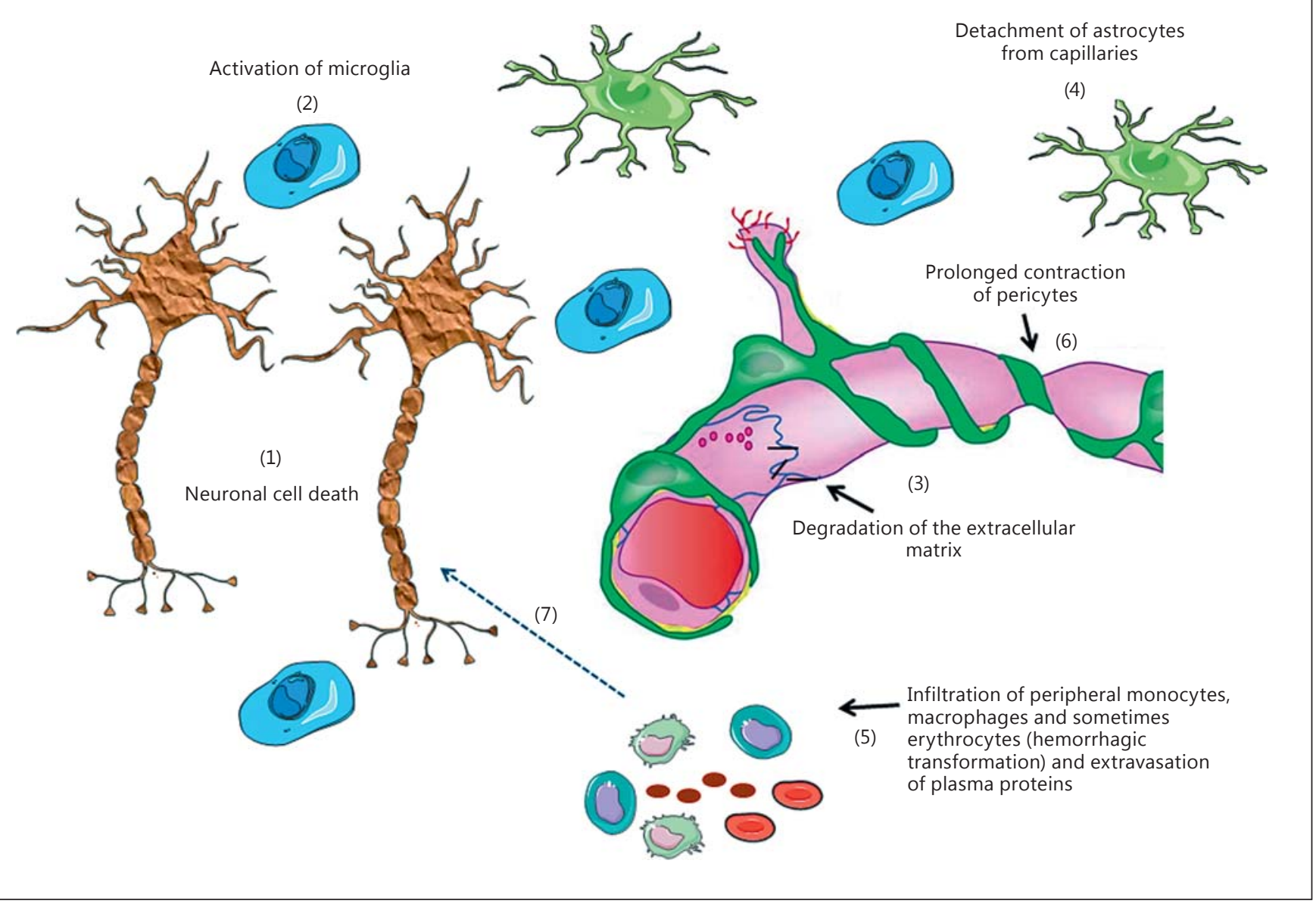

Fig. 3. Events that occur in the NVU during CI. As a consequence of a reduction in $\mathrm{CBF}$ two processes occur: first, necrosis of neurons, mainly in the core (1) that results in the release of the intracellular content into the interstitial space; this process is partially responsible for microglia activation (2). Second, the BBB becomes abnormally 'leaky', which appears as a consequence of a chain of events including degradation of the extracellular matrix (3), detachment of astrocyte end feet from the capillaries (4) and dedif- ferentiation of capillary endothelial cells with tight junction protein displacement. In some cases, this process enables protein extravasation and infiltration of peripheral macrophages/monocytes and (in some cases) erythrocytes into the brain tissue (5). In addition, some data indicate that pericytes die and contraction (rigor mortis) of their filaments (6) further impede blood flow and limits reperfusion. These processes cause additional activation of microglia (7) and may contribute to neuronal death. crovessels, microglia and astrocytes increase the generation of pro-MMP-9 and pro-MMP-2 [50, 51], resulting in up-regulation of MMP-9 (gelatinase B) and MMP-2 (gelatinase A) [48, 50, 52]. Oligodendrocyte precursor cells also secrete MMP-9 [53]. MMPs degrade extracellular matrix proteins, like type IV collagen, heparan sulfate proteoglycan, fibronectin, laminin, as well as claudin-5 and occludin. These morphological changes affect features of the endothelial layer. Detachment of BECs from the extracellular matrix caused by the activity of MMP and VEGF triggers dedifferentiation and mitotic activity in endothelial cells together with programmed cell death in some of these cells $[2,48,50,54]$. In addition, degradation of the extracellular matrix results in loss of matrix adhesion receptors [2]. Rapid loss of $\beta_{1}$-integrin receptors, which anchor endothelial cells and astrocytes to extracellular matrix proteins in the basement membrane, also occurs, resulting in leakiness of the BBB, which consequently increases the cerebrovascular permeability $[2$, 54-57]. In addition, as mentioned above, astrocytes release VEGF, which enhances dedifferentiation and mitotic activity and, thus, further increases paracellular permeability of the BBB [48]. Furthermore, detachment of astrocyte end feet and some pericytes from the basal lam- 
Table 1. Microglial markers that are considered typical for the M1 and M2 phenotypes

\begin{tabular}{ll}
\hline M1 phenotype & M2 phenotype \\
\hline CD11b & CD206 \\
CD32 & CD209 \\
CD16 & Ym-1 \\
CD23 & CD68 \\
CD40 (TNFR) & Mrc1 \\
CD86 (B7-2) & CD163 \\
CD80 (B7-1) & CD204 \\
MHC II & Fizz1 (Relma) \\
F4/80 & Dectin-1 \\
CCR7 & \\
\hline
\end{tabular}

Fizz1 = Resting-like molecule $\alpha$; CCR7 = C-C chemokine receptor type 7; F4/80 = mouse homolog of EGF-like module-containing mucin-like hormone receptor-like 1; $\mathrm{Mrc1}$ = macrophage mannose receptor, $\mathrm{C}$ type $1 ; \mathrm{Ym}-1=$ lectin gene family protein 1 .

ina was observed, which also affected integrity of the BBB (fig. 3) [50]. In addition, chemotactic factors like tumor necrosis factor (TNF)- $\alpha$ and IL-1 $\beta$, which are released from microglia and astrocytes, attract peripheral immune cells (fig. 3) [50]; this process is facilitated by altered phenotype of the BEC. Microglia and astrocytes release proinflammatory cytokines such as TNF- $\alpha$ and IL- $1 \beta$. TNF- $\alpha$ triggers endothelial cell proliferation, while both cytokines up-regulate endothelial adhesion molecule expression (e.g. ICAM-1, P-selectin and E-selectin); these two processes (cell proliferation and adhesion molecule expression), increase vascular permeability to promote extravasation of peripheral immune cells, erythrocytes, platelets and fibrin to the site of injury, which enhances inflammation (fig. 3) [58, 59].

\section{Ischemia Promptly Activates Resting Microglia and Brain Macrophages}

Several lines of evidence indicate that CI activates resting microglia and resident macrophages in the brain [60]. Activation changes their shape; they become spherical, devoid of branching processes and often contain numerous lysosomes and phagosomes. Activation is to some extent achieved by necrosis of neurons and/or other brain cells, while signaling from other cells of the NVU and protein extravasation/leukocyte infiltration across the 'leaky' BBB also play a role (fig. 3). It appears that the relative contribution of these three factors could deter- mine the abundance and the pattern of microglial activation, as discussed below. Often, activated amoeboid cells could be abundant in the core, while the majority of microglia remain ramified in the bordering areas with penumbra and in the penumbra. However, the precise role of activated microglia/macrophages (or 'reactive' microglia, a term that is sometimes used as a synonym) during CI and reperfusion is still a matter of debate. Contradictory findings exist on whether microglia/macrophages exert injurious or protective effects on other brain cells following CI; some data suggested that activation of microglia and macrophages following ischemia help in removing cell debris $[61,62]$ and promote neuronal sprouting and growth, and thus play a neuroprotective role [63]. However, in other studies, microglia/macrophage activation after CI could also cause secondary expansion of infarction possibly mediated by microglia/macrophage-mediated cytokines and chemokines, which enhance inflammation, and impairs neurogenesis and axonal regeneration $[64,65]$.

This ambiguity in the effects of microglia/macrophage activation has been partially elucidated by findings that peripheral monocytes and macrophages transform, upon stimulation, into two fairly different inflammation-related phenotypes, termed M1 and M2 [66], in a process that is referred to as 'polarization'. Consequently, activation of microglia can produce two fairly different sets of active cells; it appears that this mainly depends on the initial stimuli that cause activation. In the so-called 'classical activation', which was achieved for example by exposure to interferon (IFN) $-\gamma$, microglia differentiate into the M1 phenotype, while activation of these cells by IL- 4 and/or IL-13 induces overlapping cell surface and other phenotypic changes that are distinct from those induced by classical activation and are referred to as M2 phenotype [66].

Activation of microglia/macrophages is characterized by a change in their appearance $[67,68]$ and by proliferation, migration and release of signaling molecules that recruit peripheral immune cells, as well as by the expression of several antigens, including cluster of differentiation (CD)45, CD86 and major histocompatibility complex class II (MHC II), which attract T cells $[34,69,70]$. Transformation to the M1 phenotype (classical activation) can be induced in resting microglia by lipopolysaccharides or IFN- $\gamma$, and it is believed that it is induced by prolonged exposure to inflammatory stimuli in vivo [7174]. Characteristics of the M1 phenotype include up-regulation of proinflammatory-related surface antigens, including MHC II, CD86, CD16 and CD32 (table 1) [26, 75, 76]. Cells become spherical, retract cell processes [77], 


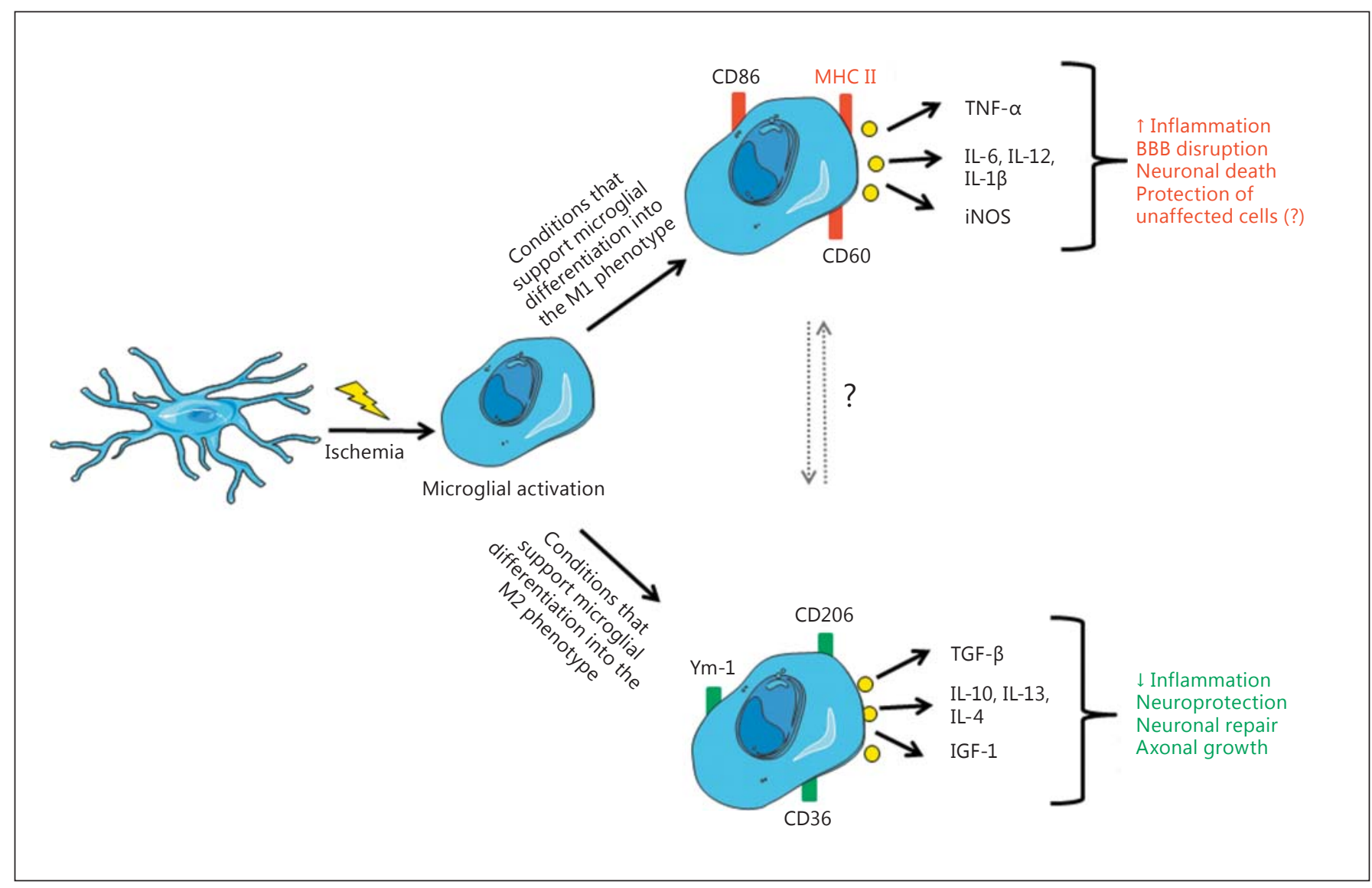

Fig. 4. Summary of microglial activation and polarization after a pathological event (e.g. CI) into the two inflammation-related phenotypes and effects of activated microglia in the ischemic brain. Precise factors that activate microglia and induce polarization into specific phenotypes remain to be identified. Also, it is not known what exactly causes the shift between the two phenotypes, which apparently occurs in vivo (indicated by a question mark). have reduced ability for phagocytosis and increase secretion of proinflammatory mediators [78], NO and proteolysis enzymes MMP-9 and MMP-3 [30, 74, 75, 79]. These processes affect basal lamina integrity and the BEC phenotype, thereby contributing to an increase in paracellular permeability of the BBB and subsequent leukocyte infiltration $[80,81]$. Secretion of proinflammatory cytokines and subsequent attraction of peripheral immune cells trigger inflammation that could cause damage to neurons and other cells of the NVU (fig. 4) [75, 76, 82, 83]. Important proinflammatory cytokines that are released under these conditions are TNF- $\alpha$, IL-12 and IL-6 $[30,74,76,79]$. Significant amount of evidence from in vitro studies suggests that activation of microglia by classical activators like IFN- $\gamma$ (i.e. polarization to the M1 phenotype) or presence of M1 phenotype microglia-derived cytokines affects the expression of tight junction proteins in the BEC monolayer and increases paracellular permeability [84].

Differentiation into the M2 phenotype can be induced in resting microglia in vitro by IL-4, IL-10 or IL-13 [71, 85, $86]$, and it is believed that it is induced in vivo by pathological events that do not cause abundant necrosis. No data exist in the literature so far about any differences between morphological features of microglia in the M1 phenotype compared to the appearance of microglia in the M2 phenotype, and therefore the differentiation between these two relies mainly on antigen expression and cytokine secretion. Microglia/macrophages with M2 phenotype express several distinctive antigens, such as Arg-1 (arginase), Ym-1 (heparin-binding lectin), CD206 (mannose receptor) and CD36 (table 1) $[26,75,76]$. A protective role of these cells is believed to be mediated by their high capability to phagocytize apoptotic and damaged 
cells, thereby preventing release of their cellular content into the extracellular space, to phagocytize various potentially injurious molecules that have been already released in the extracellular space, as well as by secreting several anti-inflammatory cytokines, including TGF- $\beta$, IL-10, IL4, IL-13 and insulin-like growth factor (fig. 4) [72, 87].

Recently, it has been stated that cells of the M2 phenotype are not homogenous in their action and expression/ secretion of receptors and cytokines; they could be subdivided into subphenotypes, often referred to as M2a, M2b and M2c $[31,88]$. The M2a phenotype is induced by IL-4, IL-5 and IL-13, and it is characterized by increased mRNA expression of Arg-1, mannose receptor and chitinase 3-like 3; it plays a role in phagocytosis, anti-inflammatory responses and repair [88]. The $\mathrm{M} 2 \mathrm{c}$ phenotype is induced by the immunosuppressive cytokines IL-10 and TGF- $\beta$, and is associated with debris scavenging and up-regulation of anti-inflammatory cytokines [31]. Contrary to that, the M2b phenotype is induced by a number of factors, including immune complexes (binding of $\mathrm{Fc} \gamma \mathrm{R}$ ) and IL- $1 \beta$ or lipopolysaccharides, and it could exert both proand anti-inflammatory effects $[35,88]$.

\section{Time Pattern of Microglia/Macrophage Activation after $\mathrm{Cl}$}

Most studies revealed that microglia do not only differentiate into one phenotype after CI; in fact, findings suggest that microglia expressing both phenotypes are present during $\mathrm{CI}$ and the recovery period, which follows $\mathrm{CI}$ if reperfusion is established $[86,87]$. However, contradictory findings exist on the relative proportion of cells that express either of the two phenotypes, on the time pattern of polarization and also on mechanisms that trigger polarization $[82,86,87]$. Bearing in mind fairly opposite effects of the two phenotypes, these have a significant relevance in understanding the role of microglia during and after CI.

Recent studies have shown that abundance of injured neurons in the tissue tends to prime microglia toward the M1 phenotype [76, 86]; this is probably due to abundant release of cellular content into the extracellular space. These findings were partially confirmed by a study that found that microglia produce proinflammatory cytokines and express M1 phenotype-specific markers within the first $24 \mathrm{~h}$ following the onset of focal CI [30].

Many other studies contradict these findings. It was found that markers of M2 were exclusively found in the core at $24 \mathrm{~h} \mathrm{[76].} \mathrm{There} \mathrm{are} \mathrm{also} \mathrm{other} \mathrm{studies} \mathrm{suggesting}$ that the M2 phenotype is a dominant one within the first few days of the recovery period, which was then followed by a gradual increase in the number of cells that activate the $\mathrm{M} 1$ phenotype. In rats, $30 \mathrm{~min}$ of middle cerebral artery occlusion induced early expression of M2 phenotype-specific markers, which was later followed by a shift to the M1 phenotype during the recovery period [86]. Microglia/macrophages with M2 phenotype features dominated in the ischemic period $24 \mathrm{~h}$ after focal CI in rats; their abundance peaked after 5 days and then declined by 14 days after ischemia, while the number of cells expressing the M1 phenotype gradually increased after ischemia and peaked after 2 weeks [86]. Expression of mRNA for TGF- $\beta$, which is a typical M2 phenotype-specific cytokine, during transient global and transient focal CI was induced $6 \mathrm{~h}$ to 2 days and $6 \mathrm{~h}$ to 7 days after ischemia, respectively $[89,90]$, data that support other in vivo studies. A study has revealed that mRNA for IL-10, which is another M2 phenotype-specific cytokine, increased progressively from $6 \mathrm{~h}$ to 7 days after permanent middle cerebral artery occlusion [91]. Recently, we found in vitro evidence indicating that oxygen-glucose deprivation (OGD) triggered early expression and secretion of M2 phenotype-specific markers and cytokines, which was followed by a mixed pattern of expression of the two phenotypes [92].

The findings mentioned above contradict each other and suggest a mixed pattern of microglia/macrophage activation that is present in the ischemic region shortly after the onset of ischemia. Also, they suggest that the time pattern of polarization and pattern of relative abundance of the two phenotypes depend on the severity and duration of ischemia or conditions that mimic ischemia.

Different patterns of mRNA expression were reported by two recent studies $[86,93]$ that examined mRNA expression of M1 and M2 phenotype markers after CI and traumatic brain injury. A study which examined markers and cytokines of microglia/macrophage M1 and M2 phenotypes from $24 \mathrm{~h}$ to 2 months after focal traumatic brain injury has found that mRNA expression for both M1 (CD86) and M2 surface markers (Ym-1 and CD68) was significantly increased 5 days after injury, while there was no significant change in the expression of Arg-1, a typical M2 phenotype-specific marker, at any time point compared to control. In the same study, it was also concluded that the responses of macrophages and microglia to focal traumatic brain injury are highest between days 3 and 7 after injury, and the response to injury resulted in a mixture of M1 and M2 phenotypes [93].
Barakat/Redzic 
Signals that induce a particular phenotype in microglia/macrophages or that cause a shift between the two phenotypes after CI remain unclear. It was found that signaling or release of cellular content from injured neurons after $\mathrm{CI}$ could trigger a shift from the M2 to the M1 phenotype [86]. In an in vitro study, signaling from other brain cells did not play a significant role in triggering activation of resting microglia/macrophages towards the M2 phenotype after OGD; this process appeared to be independent from paracrine signaling and probably initiated by expression of hypoxia-inducible factors - downstream genes in microglia [92]. However, this in vitro protocol neither produced a clear polarization of the resting microglia/macrophages towards the M1 phenotype, nor resembled the time pattern of expression of the M1 phenotype-specific markers any time pattern that was observed in vivo [92]. Bearing these findings in mind, it could be hypothesized that paracrine signaling from other cells of the NVU plays an important role for the shift between the two phenotypes and/or for polarization of resting microglia to the M1 phenotype; therefore, these processes could not be achieved in vitro in the absence of other brain cells. These findings partially resemble and partially contradict previous reports that concluded that the microenvironment plays a crucial role in triggering microglia polarization and in the 'shift' between the M1 and M2 phenotypes [66].

\section{Activated Microglia Affect Other Cells of the NVU, Though Its Effects in $\mathrm{Cl}$ Are Controversial}

It has been shown in vivo that microglia exert protective effects on neurons after focal CI [94, 95]; however, an in vitro study revealed that these protective effects were mediated either by resting microglia or polarized microglia with the M2 phenotype, while polarized microglia with the M1 phenotype exerted injurious effects on neurons in culture [90]. It has also been reported that microglia-conditioned medium was protective on astrocytes that were exposed to OGD; OGD effects were primarily mediated by glial cell line-derived neurotrophic factor (GDNF)-related expression of ERK and NF- $\mathrm{KB}$ in astrocytes $[96,97]$. Expression and secretion of GDNF by activated microglia has been well documented [98]. Other studies indicated that microglia can protect neurons and astrocytes during CI by releasing TGF- $\beta$ [95, 97]. In addition, a recent in vitro study showed that microglia-conditioned medium was protective for astrocytes that were exposed to OGD and that this effect was at least partially mediated by TGF- $\beta$ [99], a cytokine that is known to exert anti-inflammatory and neuroprotective effects in the brain [100]. It is normally present at low levels in healthy brain, but is rapidly up-regulated after any kind of brain injury, attenuating inflammatory responses by inhibiting glial proliferation, opposing action of proinflammatory cytokines and by suppressing the actions of neutrophils [100]. Several studies have shown that application of TGF- $\beta$ decreases the infarct size after focal CI $[95,97$, 100]. Another important anti-inflammatory cytokine that is also considered as a typical M2 phenotype-specific marker is IL-10; it suppresses expression and activation of the proinflammatory cytokines IL-1 and TNF- $\alpha$ and their receptors, as well as expression of Toll-like receptor $5[91,101,102]$.

Several studies have recently challenged a traditional view which assumes that M1 microglia/macrophage-derived cytokines exert predominantly injurious effects on other brain cells. Although it is known that obvious and well-documented effects of TNF- $\alpha$ in the brain include dedifferentiation of BECs and activation of apoptosis [33, 103 ], it has also been revealed that TNF- $\alpha$ and IL- $1 \beta$ exert at least some protective effect on neurons during CI [104, 105]. It was assumed that the beneficial effects of these two cytokines were at least partially mediated by activation of nerve growth factor expression [104]. It is hypothesized that the effect of TNF- $a$ largely depends on the receptor types activated by this cytokine: TNF- $\alpha$ mainly caused detrimental effects on neighboring cells if it binds to TNF receptor (TNFR) 1, while binding of TNF- $\alpha$ to TNFR2 could exert at least some neuroprotective effects [101]. TNF- $\alpha$ could also be involved in the reduction of intracellular $\left[\mathrm{Ca}^{2+}\right]$ in astrocytes, thereby reducing potential damage caused by $\mathrm{Ca}^{2+}$ overload [101]. Also, it was found that TNF- $\alpha$ activates astrocytes to produce brainderived neurotrophic factor, GDNF and VEGF, and these signaling molecules are known to exert protective effects on neurons [101], while GDNF also exerts protective effects on astrocytes by autocrine signaling [106]. Another cytokine that is released from activated microglia with M1 phenotype is IL-6 [107]. This cytokine exerts a proinflammatory effect as well as a protective effect for brain cells [108]. In fact, many studies have found that IL-6 is an anti-inflammatory cytokine $[109,110]$ playing a protective role in brain cells [111-114]. Recently, it has been reported that IL- 6 exerts protective effects on BECs during OGD [99].

We have found that in vitro all tested M1 phenotypespecific cytokines exerted protective effects on astrocytes in primary culture exposed to 24 -hour anoxia [92]. In 
fact, the most obvious was a protective effect of TNF- $\alpha$; in the presence of this cytokine, viability of astrocytes was $>90 \%$ after 24 -hour anoxia compared to around $65 \%$ during anoxia when TNF- $\alpha$ was not present in the medium; this was accompanied by a significant decrease in the percentage of cells in early and late apoptosis [92]. It could be hypothesized that these protective effects of M1 phenotype-specific cytokines on astrocytes during anoxia could play a role in supporting glial cells (i.e. astrocytes) to survive anoxic/ischemic conditions, which could be important for the proliferation and hypertrophy of these cells, a process known as gliosis.

\section{Conclusion}

Increasing evidence indicates that activated microglia play an important role in events that occur during or following CI. It appears that this pathological condition triggers polarization of microglia and resident macrophages into inflammation-related phenotypes and that patterns of polarization probably depend on the severity and dura- tion of the ischemic insult. Activated microglia/macrophages play a key role in removing tissue debris, in protecting and supporting remaining brain cells and in signaling that occur in the NVU under these conditions. However, many processes remain unclear, including exact mechanisms that activate microglia/macrophages and/or that trigger a shift between the two main phenotypes, time pattern of activation and effects of activated microglia-derived cytokines and chemokines on other cells of the NVU. A fact that these questions remain unanswered or are only partially answered is probably due to complex signaling in the NVU with secretome profiles of NVU cells during CI or anoxia not being established yet. This makes exploration of in vivo effects of microglial activation challenging. Elucidation of these processes may improve our understanding of events that occur in stroke and provide a better therapeutic approach.

\section{Disclosure Statement}

The authors declare no conflict of interests.

\section{References}

1 Arai K, Lok J, Guo S, et al: Cellular mechanisms of neurovascular damage and repair after stroke. J Child Neurol 2011;26:1193-1198.

2 Del Zoppo GJ: The neurovascular unit in the setting of stroke. J Intern Med 2010;267:156171.

3 Abbott NJ, Ronnback L, Hansson E: Astrocyte-endothelial interactions at the bloodbrain barrier. Nat Rev Neurosci 2006; 7:4153.

4 Bertini G, Bramanti P, Constantin G, et al: New players in the neurovascular unit: insights from experimental and clinical epilepsy. Neurochem Int 2013;63:652-659.

5 ElAli A, Theriault P, Rivest S: The role of pericytes in neurovascular unit remodeling in brain disorders. Int J Mol Sci 2014;15:64536474.

6 Lok J, Gupta P, Guo S, et al: Cell-cell signaling in the neurovascular unit. Neurochem Res 2007;32:2032-2045.

7 Attwell D, Buchan AM, Charpak S, et al: Glial and neuronal control of brain blood flow. Nature 2010;468:232-243.

8 Hansson E, Ronnback L: Glial neuronal signaling in the central nervous system. FASEB J 2003; 17:341-348.

9 Hall CN, Reynell C, Gesslein B, et al: Capillary pericytes regulate cerebral blood flow in health and disease. Nature 2014;508:55-60.
10 Metea MR, Newman EA: Glial cells dilate and constrict blood vessels: a mechanism of neurovascular coupling. J Neurosci 2006;26: 2862-2870.

11 Filosa JA, Bonev AD, Straub SV, et al: Local potassium signaling couples neuronal activity to vasodilation in the brain. Nat Neurosci 2006;9:1397-1403.

12 Mulligan SJ, MacVicar BA: Calcium transients in astrocyte endfeet cause cerebrovascular constrictions. Nature 2004;431:195199.

13 Blanco VM, Stern JE, Filosa JA: Tone-dependent vascular responses to astrocyte-derived signals. Am J Physiol Heart Circ Physiol 2008; 294:H2855-H2863.

14 Filosa JA, Bonev AD, Nelson MT: Calcium dynamics in cortical astrocytes and arterioles during neurovascular coupling. Circ Res 2004;95:73-81.

15 Hawkins BT, Davis TP: The blood-brain barrier/neurovascular unit in health and disease. Pharmacol Rev 2005;57:173-185.

16 Abbott NJ, Patabendige AA, Dolman DE, et al: Structure and function of the blood-brain barrier. Neurobiol Dis 2010;37:13-25.

17 Sakimoto S, Kidoya H, Naito H, et al: A role for endothelial cells in promoting the maturation of astrocytes through the apelin/APJ system in mice. Development 2012;139:13271335.
18 Brix B, Mesters JR, Pellerin L, et al: Endothelial cell-derived nitric oxide enhances aerobic glycolysis in astrocytes via HIF-1alpha-mediated target gene activation. J Neurosci 2012; 32:9727-9735.

$19 \mathrm{Mi} \mathrm{H}$, Haeberle H, Barres BA: Induction of astrocyte differentiation by endothelial cells. J Neurosci 2001;21:1538-1547.

20 Armulik A, Abramsson A, Betsholtz C: Endothelial/pericyte interactions. Circ Res 2005; 97:512-523.

21 Bonkowski D, Katyshev V, Balabanov RD, et al: The CNS microvascular pericyte: pericyteastrocyte crosstalk in the regulation of tissue survival. Fluids Barriers CNS 2011;8:8.

22 Lee HS, Han J, Bai HJ, et al: Brain angiogenesis in developmental and pathological processes: regulation, molecular and cellular communication at the neurovascular interface. FEBS J 2009;276:4622-4635.

23 Arnold T, Betsholtz C: The importance of microglia in the development of the vasculature in the central nervous system. Vasc Cell 2013; 5:4.

24 Rymo SF, Gerhardt H, Wolfhagen-Sand F, et al: A two-way communication between microglial cells and angiogenic sprouts regulates angiogenesis in aortic ring cultures. PLoS One 2011;6:e15846. 
25 Welser JV, Li L, Milner R: Microglial activation state exerts a biphasic influence on brain endothelial cell proliferation by regulating the balance of TNF and TGF- $\beta 1$. J Neuroinflammation 2010;7:89.

26 Durafourt BA, Moore CS, Zammit DA, et al: Comparison of polarization properties of human adult microglia and blood-derived macrophages. Glia 2012;60:717-727.

27 Smith JA, Das A, Ray SK, et al: Role of proinflammatory cytokines released from microglia in neurodegenerative diseases. Brain Res Bull 2012;87:10-20.

28 Tremblay ME, Stevens B, Sierra A, et al: The role of microglia in the healthy brain. J Neurosci 2011;31:16064-16069.

29 Crain JM, Nikodemova M, Watters JJ: Microglia express distinct M1 and M2 phenotypic markers in the postnatal and adult central nervous system in male and female mice. J Neurosci Res 2013;91:1143-1151.

30 Del Zoppo GJ: Inflammation and the neurovascular unit in the setting of focal cerebral ischemia. Neuroscience 2009;158:972-982.

31 Luo XG, Chen SD: The changing phenotype of microglia from homeostasis to disease. Transl Neurodegener 2012;1:9.

32 Becher B, Prat A, Antel JP: Brain-immune connection: immuno-regulatory properties of CNS-resident cells. Glia 2000;29:293304.

33 Lai AY, Todd KG: Microglia in cerebral ischemia: molecular actions and interactions. Can J Physiol Pharmacol 2006;84:49-59.

34 Kettenmann H, Hanisch UK, Noda M, et al: Physiology of microglia. Physiol Rev 2011;91: 461-553.

35 Girard S, Brough D, Lopez-Castejon G, et al: Microglia and macrophages differentially modulate cell death after brain injury caused by oxygen-glucose deprivation in organotypic brain slices. Glia 2013;61:813-824.

36 Smith JA, Das A, Ray SK, et al: Role of proinflammatory cytokines released from microglia in neurodegenerative diseases. Brain Res Bull 2012;87:10-20.

37 Pascual O, Ben Achour S, Rostaing P, et al Microglia activation triggers astrocyte-mediated modulation of excitatory neurotransmission. Proc Natl Acad Sci USA 2012;109:E197E205.

38 Eyo UB, Wu LJ: Bidirectional microglia-neuron communication in the healthy brain Neural Plast 2013;2013:456857.

39 Hung J, Chansard M, Ousman SS, et al: Activation of microglia by neuronal activity: results from a new in vitro paradigm based on neuronal-silicon interfacing technology. Brain Behav Immun 2010;24:31-40.

40 Kierdorf K, Prinz M: Factors regulating microglia activation. Front Cell Neurosci 2013; $7: 44$

41 Shih AY, Fernandes HB, Choi FY, et al: Policing the police: astrocytes modulate microglial activation. J Neurosci 2006;26:3887-3888.
42 Ferrer I, Planas AM: Signaling of cell death and cell survival following focal cerebral ischemia: life and death struggle in the penumbra. J Neuropathol Exp Neurol 2003;62:329-339.

43 Bodalia A, Li H, Jackson MF: Loss of endoplasmic reticulum $\mathrm{Ca}^{2+}$ homeostasis: contribution to neuronal cell death during cerebral ischemia. Acta Pharmacol Sin 2013;34:49-59.

44 Nakka VP, Gusain A, Mehta SL, et al: Molecular mechanisms of apoptosis in cerebral ischemia: multiple neuroprotective opportunities. Mol Neurobiol 2008;37:7-38.

45 Broughton BR, Reutens DC, Sobey CG: Apoptotic mechanisms after cerebral ischemia. Stroke 2009;40:e331-e339.

46 Baker WB, Sun Z, Hiraki T, et al: Neurovascular coupling varies with level of global cerebral ischemia in a rat model. J Cereb Blood Flow Metab 2013;33:97-105.

47 Myllyharju J: Prolyl 4-hydroxylases, master regulators of the hypoxia response. Acta Physiol (Oxf) 2013;208:148-165.

48 Arai K, Jin G, Navaratna D, Lo EH: Brain angiogenesis in developmental and pathological processes: neurovascular injury and angiogenic recovery after stroke. FEBS J 2009;276: 4644-4652.

49 Vallon M, Chang J, Zhang H, et al: Developmental and pathological angiogenesis in the central nervous system. Cell Mol Life Sci 2014;71:3489-3506.

50 Del Zoppo GJ: Toward the neurovascular unit. A journey in clinical translation: 2012 Thomas Willis Lecture. Stroke 2013;44:263269.

51 Del Zoppo GJ, Frankowski H, Gu YH, et al: Microglial cell activation is a source of metalloproteinase generation during hemorrhagic transformation. J Cereb Blood Flow Metab 2012;32:919-932.

52 Rock RB, Gekker G, Hu S, et al: Role of microglia in central nervous system infections. Clin Microbiol Rev 2004;17:942-964.

53 Seo JH, Miyamoto N, Hayakawa K, et al: Oligodendrocyte precursors induce early bloodbrain barrier opening after white matter injury. J Clin Invest 2013;123:782-786.

54 Del Zoppo GJ, Mabuchi T: Cerebral microvessel responses to focal ischemia. J Cereb Blood Flow Metab 2003;23:879-894.

55 Del Zoppo GJ, Milner R, Mabuchi T, et al: Vascular matrix adhesion and the bloodbrain barrier. Biochem Soc Trans 2006;34: 1261-1266.

56 Del Zoppo GJ, Milner R: Integrin-matrix interactions in the cerebral microvasculature. Arterioscler Thromb Vasc Biol 2006;26: 1966-1975.

57 Zhang L, Zhang ZG, Chopp M: The neurovascular unit and combination treatment strategies for stroke. Trends Pharmacol Sci 2012;33: 415-422.

58 Moskowitz MA, Lo EH, Iadecola C: The science of stroke: mechanisms in search of treatments. Neuron 2010;67:181-198.
59 Woodruff TM, Thundyil J, Tang SC, et al: Pathophysiology, treatment, and animal and cellular models of human ischemic stroke. Mol Neurodegener 2011;6:11.

60 Boscia F, D'Avanzo C, Pannaccione A, et al: New roles of NCX in glial cells: activation of microglia in ischemia and differentiation of oligodendrocytes. Adv Exp Med Biol 2013; 961:307-316.

61 Lalancette-Hebert M, Gowing G, Simard A, et al: Selective ablation of proliferating microglial cells exacerbates ischemic injury in the brain. J Neurosci 2007;27:2596-2605.

62 Hanisch UK, Kettenmann H: Microglia: active sensor and versatile effector cells in the normal and pathologic brain. Nat Neurosci 2007;10:1387-1394.

63 Kigerl KA, Gensel JC, Ankeny DP, et al: Identification of two distinct macrophage subsets with divergent effects causing either neurotoxicity or regeneration in the injured mouse spinal cord. J Neurosci 2009;29:13435-13444.

64 Ekdahl CT, Claasen JH, Bonde S, et al: Inflammation is detrimental for neurogenesis in adult brain. Proc Natl Acad Sci USA 2003;100: 13632-13637.

65 Schwab ME, Bartholdi D: Degeneration and regeneration of axons in the lesioned spinal cord. Physiol Rev 1996;76:319-370.

66 Gordon S: Alternative activation of macrophages. Nat Rev Immunol 2003;3:23-35.

67 Ponomarev ED, Veremeyko T, Weiner HL: MicroRNAs are universal regulators of differentiation, activation, and polarization of $\mathrm{mi}$ croglia and macrophages in normal and diseased CNS. Glia 2013;61:91-103.

68 Walker FR, Nilsson M, Jones K: Acute and chronic stress-induced disturbances of microglial plasticity, phenotype and function. Curr Drug Targets 2013;14:1262-1276.

69 Aloisi F: Immune function of microglia. Glia 2001;36:165-179.

70 Kofler J, Wiley CA: Microglia: key innate immune cells of the brain. Toxicol Pathol 2011; 39:103-114.

71 Girard S, Brough D, Lopez-Castejon G, et al: Microglia and macrophages differentially modulate cell death after brain injury caused by oxygen-glucose deprivation in organotypic brain slices. Glia 2013;61:813-824.

72 Guedes J, Cardoso AL, Pedroso de Lima MC: Involvement of microRNA in microglia-mediated immune response. Clin Dev Immunol 2013;2013:186872.

73 Harry GJ: Microglia during development and aging. Pharmacol Ther 2013;139:313-326.

74 Michelucci A, Heurtaux T, Grandbarbe L, et al: Characterization of the microglial phenotype under specific pro-inflammatory and anti-inflammatory conditions: effects of oligomeric and fibrillar amyloid-beta. J Neuroimmunol 2009;210:3-12.

75 Patel AR, Ritzel R, McCullough LD, et al: Microglia and ischemic stroke: a double-edged sword. Int J Physiol Pathophysiol Pharmacol 2013;5:73-90. 
76 Taylor RA, Sansing LH: Microglial responses after ischemic stroke and intracerebral hemorrhage. Clin Dev Immunol 2013;2013: 746068.

77 Boche D, Perry VH, Nicoll JA: Review: activation patterns of microglia and their identification in the human brain. Neuropathol Appl Neurobiol 2013;39:3-18.

78 Fairweather D, Cihakova D: Alternatively activated macrophages in infection and autoimmunity. J Autoimmun 2009;33:222-230.

79 Olah M, Biber K, Vinet J, et al: Microglia phenotype diversity. CNS Neurol Disord Drug Targets 2011;10:108-118.

80 Corti R, Hutter R, Badimon JJ, et al: Evolving concepts in the triad of atherosclerosis, inflammation and thrombosis. J Thromb Thrombolysis 2004;17:35-44.

81 Zameer A, Hoffman SA: Increased ICAM-1 and VCAM-1 expression in the brains of autoimmune mice. J Neuroimmunol 2003;142: 67-74.

82 Huang YC, Feng ZP: The good and bad of microglia/macrophages: new hope in stroke therapeutics. Acta Pharmacol Sin 2013;34:67.

83 Spitzbarth I, Baumgartner W, Beineke A: The role of pro- and anti-inflammatory cytokines in the pathogenesis of spontaneous canine CNS diseases. Vet Immunol Immunopathol 2012 15;147:6-24.

84 da Fonseca AC, Matias D, Garcia C, et al: The impact of microglial activation on bloodbrain barrier in brain diseases. Front Cell Neurosci 2014;8:362

85 Cherry JD, Olschowka JA, O'Banion MK: Neuroinflammation and M2 microglia: the good, the bad, and the inflamed. J Neuroinflammation 2014;11:98.

$86 \mathrm{Hu}$ X, Li P, Guo Y, et al: Microglia/macrophage polarization dynamics reveal novel mechanism of injury expansion after focal cerebral ischemia. Stroke 2012;43:30633770.

87 Jin R, Yang G, Li G: Inflammatory mechanisms in ischemic stroke: role of inflammatory cells. J Leukoc Biol 2010;87:779-789.

88 David S, Kroner A: Repertoire of microglial and macrophage responses after spinal cord injury. Nat Rev Neurosci 2011;12:388-399.

89 Lehrmann E, Kiefer R, Finsen B, et al: Cytokines in cerebral ischemia: expression of transforming growth factor beta-1 (TGF-beta 1) mRNA in the postischemic adult rat hippocampus. Exp Neurol 1995;131:114123.
90 Zhu Y, Roth-Eichhorn S, Braun N, et al: The expression of transforming growth factorbeta1 (TGF-beta1) in hippocampal neurons: a temporary upregulated protein level after transient forebrain ischemia in the rat. Brain Res 2000;866:286-298.

91 Perez-de Puig I, Miro F, Salas-Perdomo A, et al: IL-10 deficiency exacerbates the brain inflammatory response to permanent ischemia without preventing resolution of the lesion. J Cereb Blood Flow Metab 2013;33: 1955-1966.

92 Barakat R, Redzic Z: Differential cytokine expression by brain microglia/macrophages in primary culture after oxygen glucose deprivation and their protective effects on astrocytes during anoxia. Fluids Barriers CNS 2015;12:6.

93 Turtzo LC, Lescher J, Janes L, et al: Macrophagic and microglial responses after focal traumatic brain injury in the female rat. J Neuroinflammation 2014;11:82.

94 Imai F, Suzuki H, Oda J, et al: Neuroprotective effect of exogenous microglia in global brain ischemia. J Cereb Blood Flow Metab 2007;27:488-500.

95 Lee GA, Lin CH, Jiang HH, et al: Microgliaderived glial cell line-derived neurotrophic factor could protect Sprague-Dawley rat astrocyte from in vitro ischemia-induced damage. Neurosci Lett 2004;356:111-114.

96 Chu LF, Wang WT, Ghanta VK, et al: Ischemic brain cell-derived conditioned medium protects astrocytes against ischemia through GDNF/ERK/NF- $\kappa B$ signaling pathway. Brain Res 2008;1239:24-35.

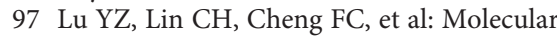
mechanisms responsible for microglia-derived protection of Sprague-Dawley rat brain cells during in vitro ischemia. Neurosci Lett 2005;373:159-164.

98 Wang G, Zhang J, Hu X, et al: Microglia/ macrophage polarization dynamics in white matter after traumatic brain injury. J Cereb Blood Flow Metab 2013;33:1864-1874.

99 Redzic ZB, Rabie T, Sutherland BA, et al: Differential effects of paracrine factors on the survival of cells of the neurovascular unit during oxygen glucose deprivation. Int J Stroke 2015;10:407-414.

100 Goss CE, Bednar MM, Howard DB, et al: Transforming growth factor-beta 1 reduces infarct size after experimental cerebral ischemia in a rabbit model. Stroke 1993;24:558562.

101 Ceulemans AG, Zgavc T, Kooijman R, et al: The dual role of the neuroinflammatory response after ischemic stroke: modulatory effects of hypothermia. J Neuroinflammation 2010;7:74
102 Lakhan SE, Kirchgessner A, Hofer M: Inflammatory mechanisms in ischemic stroke: therapeutic approaches. J Transl Med 2009; 7:97.

103 Watters O, O'Connor JJ: A role for tumor necrosis factor-alpha in ischemia and ischemic preconditioning. J Neuroinflammation $2011 ; 8: 87$

104 Kadhim HJ, Duchateau J, Sebire G: Cytokines and brain injury: invited review. J Intensive Care Med 2008;23:236-249.

105 Wang Q, Tang XN, Yenari MA: The inflammatory response in stroke. J Neuroimmunol 2007; 184:53-68.

106 Sandhu JK, Gardaneh M, Iwasiow R, et al: Astrocyte-secreted GDNF and glutathione antioxidant system protect neurons against 6OHDA cytotoxicity. Neurobiol Dis 2009; 33:405-414.

107 Rincon M: Interleukin-6: from an inflammatory marker to a target for inflammatory diseases. Trends Immunol 2012;33:571577.

108 Scheller J, Chalaris A, Schmidt-Arras D, et al: The pro- and anti-inflammatory properties of the cytokine interleukin-6. Biochim Biophys Acta 2011;1813:878-888.

109 Tilg H, Trehu E, Atkins MB, et al: Interleukin-6 (IL-6) as an anti-inflammatory cytokine: induction of circulating IL-1 receptor antagonist and soluble tumor necrosis factor receptor p55. Blood 1994;83:113-118.

110 Xing Z, Gauldie J, Cox G, et al: IL-6 is an antiinflammatory cytokine required for controlling local or systemic acute inflammatory responses. J Clin Invest 1998;101:311320.

111 Ali C, Nicole O, Docagne F, et al: Ischemiainduced interleukin- 6 as a potential endogenous neuroprotective cytokine against NMDA receptor-mediated excitotoxicity in the brain. J Cereb Blood Flow Metab 2000; 20:956-966

112 Loddick SA, Turnbull AV, Rothwell NJ: Cerebral interleukin- 6 is neuroprotective during permanent focal cerebral ischemia in the rat. J Cereb Blood Flow Metab 1998;18:176179.

113 Yamashita T, Sawamoto K, Suzuki S, et al: Blockade of interleukin-6 signaling aggravates ischemic cerebral damage in mice: possible involvement of Stat 3 activation in the protection of neurons. J Neurochem 2005; 94:459-468.

114 Hall C, Reynell C, Gesslein B, et al: Capillary pericytes regulate cerebral blood flow in health and disease. Nature 2014;508:55-60. 